\title{
ON LIE ALGEBRAS IN BRAIDED CATEGORIES
}

\author{
BODO PAREIGIS \\ Mathematisches Institut der Universität München \\ Theresienstr. 39, 80333 Munich, Germany \\ E-mail: pareigis@rz.mathematik.uni-muenchen.de
}

Herrn Prof. Dr. Friedrich Kasch zum 75. Geburtstag gewidmet

\begin{abstract}
The category of group-graded modules over an abelian group $G$ is a monoidal category. For any bicharacter of $G$ this category becomes a braided monoidal category. We define the notion of a Lie algebra in this category generalizing the concepts of Lie super and Lie color algebras. Our Lie algebras have $n$-ary multiplications between various graded components. They possess universal enveloping algebras that are Hopf algebras in the given category. Their biproducts with the group ring are noncommutative noncocommutative Hopf algebras some of them known in the literature. Conversely the primitive elements of a Hopf algebra in the category form a Lie algebra in the above sense.
\end{abstract}

1. Introduction. With the appearance of quantum groups the study of Hopf algebras has taken an important turn in recent years. Many families of quantum groups are known. Here we will add some new families by means of a construction which might eventually also help to develop a structure theory of quantum groups. In classical structure theory a formal group is decomposed into a smash product of an infinitesimal part with a separable part. The separable part is often a group algebra, whereas the infinitesimal part is often a universal enveloping algebra on which the group algebra operates. The infinitesimal part then is generated by the primitive elements of the formal group.

However, for quantum groups or noncommutative noncocommutative Hopf algebras this kind of decomposition seems to be much more complicated. We will use the following approach.

Let $\chi: G \times G \longrightarrow k^{*}$ be a bicharacter of the abelian group $G$. Then the monoidal category of $G$-graded vector spaces or $k G$-comodules is a braided monoidal category.

1991 Mathematics Subject Classification: Primary 16W30, 17B70; Secondary 16W55, 16S30, $16 \mathrm{~S} 40$

Key words and phrases: graded Lie algebra, braided category, braided Hopf algebra, universal enveloping algebra.

The paper is in final form and no version of it will be published elsewhere. 
By forming a biproduct with the group algebra $k G$, each Hopf algebra in this category generates an ordinary Hopf algebra. This process was studied for Hopf algebras with a projection by Radford in [R] (or as the process of bosonization by Majid in [M94a]).

We will give some general techniques for constructing Hopf algebras in the category of $G$-graded vector spaces. Our special interest lies in Hopf algebras that are generated by primitive elements in the proper sense, that is $\Delta(x)=x \otimes 1+1 \otimes x$. In the biproduct these elements become skew primitive elements.

There is a generalized notion of a Lie algebra in symmetric monoidal categories under the name of Lie color algebra (or Lie super algebra). An appropriate definition of a Lie algebra in a braided monoidal category should have specific properties. In the classical situation the primitive elements of a Hopf algebra form a Lie algebra. Also the set of derivations of an algebra is a Lie algebra. So a more general definition of a Lie algebra should pass a test with respect to the set primitive elements of a Hopf algebra and with respect to the set of derivations of an algebra. We propose a definition of generalized Lie algebras in the braided monoidal category of $G$-graded vector spaces. These generalized Lie algebras have $n$-ary bracket multiplications that are only partially defined, but certain symmetry and Jacobi identities still hold.

Lie color and Lie super algebras as well as ordinary Lie algebras are special cases of our generalized Lie algebras. We will show that the set of primitive elements of a Hopf algebra is such a generalized Lie algebra. Every associative algebra is also a generalized Lie algebra by the same definition of the bracket multiplication. And the set of derivations of an algebra will turn out to be a Lie algebra.

Starting out with a generalized Lie algebra we will construct a universal enveloping algebra that turns out to be a Hopf algebra in the category of $G$-graded vector spaces. Thus we have obtained a method for constructing new Hopf algebras in this category. By forming biproducts we obtain many old and new ordinary noncommutative noncocommutative Hopf algebras or quantum groups.

Ordinary Hopf algebras over a field of characteristic zero that are generated by their primitive elements are either trivial or infinite dimensional. In fact they are universal enveloping algebras of Lie algebras.

Hopf algebras in braided monoidal categories in characteristic zero that are generated by their primitive elements, however, do not share this property. In certain cases they are similar to universal restricted enveloping algebras of restricted (p-)Lie algebras.

A simple example of such a Hopf algebra is $\mathbf{C}[x] /\left(x^{n}\right)$ with $\Delta(x)=x \otimes 1+1 \otimes x$ considered as an object in the braided monoidal category of $C_{n}$-graded vector spaces, where $C_{n}$ is the cyclic group of order $n$ and the braiding is given by a primitive $n$-th root of unity. It is generated by the primitive element $x$ as an algebra in the given category, but it is finite dimensional. Such a Hopf algebra cannot exist as an ordinary Hopf algebra (in the category of vector spaces).

We will restrict our considerations to group graded vector spaces over a fixed abelian group $G$. A consequence of one of our main results is that for primitive elements $x_{1}, \ldots, x_{n}$ 
of degree 1 in a $C_{n}$-graded Hopf algebra $H$ the expression

$$
\left[x_{1}, \ldots, x_{n}\right]:=\sum_{\sigma \in S_{n}} x_{\sigma(1)} \ldots x_{\sigma(n)}
$$

is again a primitive element (of degree zero) in $H$ (where $S_{n}$ is the symmetric group).

By now the reader should be interested to see the definition of a generalized $G$ graded Lie algebra. Let $G$ be an abelian group with a bicharacter $\chi: G \times G \longrightarrow k^{*}$. For every primitive $n$-th root of unity $\zeta$ we consider certain $n$-tuples $\left(g_{1}, \ldots, g_{n}\right)$ in $G$ associated with $\zeta$. We will call them $\zeta$-families. Furthermore we construct factors $\rho\left(\sigma,\left(g_{1}, \ldots, g_{n}\right)\right) \in k^{*}$ for each permutation $\sigma \in S_{n}$ and each $\zeta$-family $\left(g_{1}, \ldots, g_{n}\right)$. These factors generalize the sign of a permutation. Details will be given in Definition 2.1. A generalized $G$-graded Lie algebra is a $G$-graded vector space $P=\bigoplus_{g \in G} P_{g}$ that has multilinear bracket operations for all $\zeta$ and all $\zeta$-families $\left(g_{1}, \ldots, g_{n}\right)$

$$
[\ldots]: P_{g_{1}} \times \ldots \times P_{g_{n}} \longrightarrow P_{g_{1}+\ldots+g_{n}}
$$

satisfying the following generalizations of the symmetry and Jacobi identities

- $\left[x_{1}, \ldots, x_{n}\right]=\rho\left(\sigma,\left(g_{1}, \ldots, g_{n}\right)\right)\left[x_{\sigma(1)}, \ldots, x_{\sigma(n)}\right]$,

- $\sum_{i=1}^{n+1} \zeta^{-i+1}\left(\prod_{j=1}^{i-1} \chi\left(g_{j}, g_{i}\right)\right)\left[x_{i},\left[x_{1}, \ldots, \hat{x}_{i}, \ldots, x_{n+1}\right]\right]=0$,

- $\left[x,\left[y_{1}, y_{2}, \ldots, y_{n}\right]\right]=\sum_{i=1}^{n}\left(\prod_{j=1}^{i-1} \chi\left(g_{j}, h\right)\right)\left[y_{1}, \ldots,\left[x, y_{i}\right], \ldots, y_{n}\right]$

whenever the bracket products are defined. In particular we require $x_{i}, y_{i} \in P_{g_{i}}$ and $x \in P_{h}$.

The set of primitive elements of a $G$-graded Hopf algebra is an example for a generalized Lie algebra, and so is any $G$-graded algebra if we define the bracket operation by

$$
\left[x_{1}, \ldots, x_{n}\right]:=\sum_{\sigma \in S_{n}} \rho\left(\sigma,\left(g_{1}, \ldots, g_{n}\right)\right) x_{\sigma(1)} \cdot \ldots \cdot x_{\sigma(n)} .
$$

A Lie super algebra $\left(P_{0}, P_{1}\right)$, where $P_{0}$ is an ordinary Lie algebra with operations $[.,]:. P_{1} \otimes P_{1} \longrightarrow P_{0}$ and [.,.] : $P_{0} \otimes P_{1} \longrightarrow P_{1}$, is an example for this definition. Lie color algebras are also special cases of our concept.

I would like to acknowledge helpful conversations with Edward L. Green, Frank Halanke, Susan Montgomery, Martin Neuchl, Helmut Rohrl, Peter Schauenburg, and Yorck Sommerhäuser.

2. Bicharacters and the bracket multiplication. Throughout let $k$ be an integral domain, let $G$ be an abelian group written additively and let $\chi: G \times G \longrightarrow k^{*}$ be a bicharacter, that is a group homomorphism $\chi: G \otimes \mathbf{Z} G \longrightarrow k^{*}$. Then bicharacters on $\mathbf{Z}$ resp. $\mathbf{Z} / n \mathbf{Z}$ have the form $\chi(i, j)=\zeta^{i j}$.

Definition 2.1. Let $\zeta \in k^{*}$. An $n$-tuple $\left(g_{1}, \ldots, g_{n}\right)$ of (not necessarily distinct) elements $g_{i} \in G$ is a $\zeta$-family of length $n$, if

$$
\chi\left(g_{i}, g_{j}\right) \chi\left(g_{j}, g_{i}\right)=\zeta^{2}
$$

for all $i \neq j$.

Observe that a $\zeta$-family $\left(g_{1}, \ldots, g_{n}\right)$ is also a $(-\zeta)$-family. 
If $\left(g_{1}, \ldots, g_{n}\right)$ is a $\zeta$-family and $\sigma \in S_{n}$ then $\sigma\left(g_{1}, \ldots, g_{n}\right):=\left(g_{\sigma(1)}, \ldots, g_{\sigma(n)}\right)$ is also a $\zeta$-family. Thus the set $G_{n}^{\zeta}$ of all $\zeta$-families of length $n$ is an $S_{n}$-set. Observe that there are $\zeta$-families of varying lengths $n$.

Let $X \subset G_{n}^{\zeta}$ be an $S_{n}$-closed subset. We define a map $\rho: S_{n} \times X \longrightarrow k^{*}$ by

$$
\rho\left(\sigma,\left(g_{1}, \ldots, g_{n}\right)\right):=\prod_{(i, j) \in R}\left(\zeta^{-1} \chi\left(g_{\sigma(j)}, g_{\sigma(i)}\right)\right)
$$

where $R$ is the set of all pairs $(i, j)$ with $1 \leq i<j \leq n$ and $\sigma(i)>\sigma(j)$, i.e. the set of all pairs in $\sigma$ in reverse position.

LEMMA 2.2. The map $\rho$ satisfies the following relation

$$
\rho\left(\sigma \tau,\left(g_{1}, \ldots, g_{n}\right)\right)=\rho\left(\tau,\left(g_{\sigma(1)}, \ldots, g_{\sigma(n)}\right)\right) \rho\left(\sigma,\left(g_{1}, \ldots, g_{n}\right)\right) .
$$

Proof. We have to show

$$
\begin{aligned}
& \prod_{i<j, \sigma \tau(i)>\sigma \tau(j)}\left(\zeta^{-1} \chi\left(g_{\sigma \tau(j)}, g_{\sigma \tau(i)}\right)\right) \\
& =\left(\prod_{k<l, \tau(k)>\tau(l)}\left(\zeta^{-1} \chi\left(g_{\sigma \tau(l)}, g_{\sigma \tau(k)}\right)\right)\right)\left(\prod_{r<s, \sigma(r)>\sigma(s)}\left(\zeta^{-1} \chi\left(g_{\sigma(s)}, g_{\sigma(r)}\right)\right)\right) .
\end{aligned}
$$

We investigate the factors on the left hand side. If $i<j$ with $\sigma \tau(i)<\sigma \tau(j)$ then there is no corresponding factor on the l.h.s. On the r.h.s. there are now two possibilities. If $s:=\tau(i)>\tau(j)=: r$ (and thus $\sigma(r)>\sigma(s))$ then the first product contributes a factor $\zeta^{-1} \chi\left(g_{\sigma \tau(j)}, g_{\sigma \tau(i)}\right)$ for the pair $i<j, \tau(i)>\tau(j)$ and the second product contributes a factor $\zeta^{-1} \chi\left(g_{\sigma \tau(i)}, g_{\sigma \tau(j)}\right)$ for the pair $r<s, \sigma(r)>\sigma(s)$. These two factors cancel. If $s:=\tau(i)<\tau(j)=$ : $r$ then neither pair $i<j, \tau(i)<\tau(j)$ nor $r<s, \sigma(r)<\sigma(s)$ contributes a factor.

However, if $i<j$ with $\sigma \tau(i)>\sigma \tau(j)$ then there is a corresponding factor $\zeta^{-1} \chi\left(g_{\sigma \tau(j)}, g_{\sigma \tau(i)}\right)$ on the l.h.s. On the r.h.s. there are again two possibilities. If $\tau(i)>$ $\tau(j)$ then the first product contributes a factor $\zeta^{-1} \chi\left(g_{\sigma \tau(j)}, g_{\sigma \tau(i)}\right)$ for the pair $i<$ $j, \tau(i)>\tau(j)$. The second product does not contribute a factor for the pair $s:=\tau(i)>$ $\tau(j)=: r, \sigma(r)<\sigma(s)$. If $s:=\tau(i)<\tau(j)=: r$ then the first product does not contribute a factor for the pair $i<j, \tau(i)<\tau(j)$, but the second product contributes a factor $\zeta^{-1} \chi\left(g_{\sigma \tau(i)}, g_{\sigma \tau(j)}\right)$ for the pair $r<s, \sigma(r)>\sigma(s)$. This argument takes care of all factors on both sides of the formula.

If $g, g^{\prime} \in G$ have order $m$ resp. $m^{\prime}$ and if $n=\operatorname{gcd}\left(m, m^{\prime}\right)$ then $\chi\left(g, g^{\prime}\right)^{n}=1$ since $\chi\left(g, g^{\prime}\right)^{m}=\chi\left(m g, g^{\prime}\right)=\chi\left(0, g^{\prime}\right)=1$ and $\chi\left(g, g^{\prime}\right)^{m^{\prime}}=\chi\left(g, m^{\prime} g^{\prime}\right)=1$. Hence $\chi\left(g, g^{\prime}\right)$ is a primitive $n$-th root of unity for all $g, g^{\prime} \in G$ of finite order, with $n$ chosen suitably.

Apart from this restriction on the choice of $\chi\left(g, g^{\prime}\right)$ any combination of values can occur. In particular let $\zeta$ be an $n$-th root of unity. Then there are examples of groups $G$ and elements $g_{1}, \ldots, g_{m} \in G$ with $\chi\left(g_{i}, g_{j}\right)=\zeta$ for all $i \neq j$. Take for example $G=C_{n} \oplus \ldots \oplus C_{n}=\mathbf{Z} g_{1} \oplus \ldots \oplus \mathbf{Z} g_{m}$. Then a bicharacter $\chi$ is defined as the group homomorphism $\chi: G \otimes G=\bigoplus_{i, j=1}^{m} \mathbf{Z} g_{i} \otimes \mathbf{Z} g_{j}=\bigoplus_{i, j=1}^{m} \mathbf{Z}\left(g_{i} \otimes g_{j}\right) \longrightarrow k^{*}$, where the images $\chi\left(g_{i} \otimes g_{j}\right)=\chi\left(g_{i}, g_{j}\right)$ are chosen arbitrarily among the $n$-th roots of unity (including 1). 
If $\left(g_{1}, \ldots, g_{n}\right)$ is a $\zeta$-family with at least one element $g_{i}$ of finite order, then $\chi\left(g_{i}, g_{j}\right) \chi\left(g_{j}, g_{i}\right)=\zeta^{2}$ implies that $\zeta$ is a root of unity.

ExAmple 2.3. For $g \in G$ we define $|g|:=\chi(g, g)$. If $g$ has order $m$ then $|g|$ is a primitive $n$-th root of unity where $n$ divides the order $m$ of $g$. Furthermore $(g, \ldots, g)$ is a $|g|$-family in $G$. Observe also that any pair $(0, g)$ or $(g, 0)$ is a 1 -family and a $(-1)$-family.

The map $\rho: S_{n} \times X \longrightarrow k^{*}$ reduces to a well known map in the following situation. If $\left(g_{1}, \ldots, g_{n}\right)=(g, \ldots, g),|g|=1$, and $\zeta=-1$ then $\rho(\sigma,(g, \ldots, g))=\operatorname{sgn}(\sigma)$ is the sign of the permutation.

Now we turn to the category of interest for us. By [FM] Remark 3.4 the category $\mathcal{M}^{k G}$ of $G$-graded $k$-modules (or the category of $k G$-comodules) is a braided monoidal category with the tensor product

$$
(X \otimes Y)_{g}=\bigoplus_{h \in G} X_{h} \otimes Y_{g-h}
$$

and the braiding

$$
\tau: X \otimes Y \ni x \otimes y \mapsto \chi(\operatorname{deg}(x), \operatorname{deg}(y)) y \otimes x \in Y \otimes X
$$

where $x$ and $y$ are homogeneous elements of degree $\operatorname{deg}(x), \operatorname{deg}(y) \in G$.

Algebras, coalgebras, bialgebras, and Hopf algebras in the braided monoidal category $\mathcal{M}^{k G}$ will be called $(G, \chi)$-algebras, $(G, \chi)$-coalgebras, $(G, \chi)$-bialgebras, resp. $(G, \chi)$ Hopf algebras.

Let $A, B$ be $(G, \chi)$-algebras in the category $\mathcal{M}^{k G}$. Then $A \otimes B$ is also a $(G, \chi)$-algebra in $\mathcal{M}^{k G}$ with the multiplication $A \otimes B \otimes A \otimes B \stackrel{1 \otimes \tau \otimes 1}{\longrightarrow} A \otimes A \otimes B \otimes B \stackrel{m_{A} \otimes m_{B}}{\longrightarrow} A \otimes B$ (see for example [M94b]).

Definition 2.4. Let $A$ be a $(G, \chi)$-algebra (associative with unit) in $\mathcal{M}^{k G}$. We define

$$
\left[x_{1}, \ldots, x_{n}\right]:=\sum_{\sigma \in S_{n}} \rho\left(\sigma,\left(g_{1}, \ldots, g_{n}\right)\right) x_{\sigma(1)} \cdot \ldots \cdot x_{\sigma(n)}
$$

for all $\zeta \in k^{*}$, all $\zeta$-families $\left(g_{1}, \ldots, g_{n}\right)$, and all $x_{i} \in A_{g_{i}}$.

A special example is $\zeta=-1, n=2$ and $\chi\left(g_{1}, g_{2}\right)=\chi\left(g_{2}, g_{1}\right)=1$. Then

$$
\left[x_{1}, x_{2}\right]=x_{1} x_{2}+\rho\left((2,1),\left(g_{1}, g_{2}\right)\right) x_{2} x_{1}=x_{1} x_{2}-x_{2} x_{1} .
$$

If $\zeta=-1, n=2$ and $\chi\left(g_{1}, g_{2}\right)=\chi\left(g_{2}, g_{1}\right)=-1$, then

$$
\left[x_{1}, x_{2}\right]=x_{1} x_{2}+\rho\left((2,1),\left(g_{1}, g_{2}\right)\right) x_{2} x_{1}=x_{1} x_{2}+x_{2} x_{1} .
$$

Hence we obtain Lie algebras resp. Lie super algebras as a special case of (2.4). Similarly if $\zeta=-1, n=2$ and $\left(g_{1}, g_{2}\right)$ is a $\zeta$-family then $\chi\left(g_{1}, g_{2}\right)=\chi\left(g_{2}, g_{1}\right)^{-1}$ and

$$
\left[x_{1}, x_{2}\right]=x_{1} x_{2}-\chi\left(g_{1}, g_{2}\right) x_{2} x_{1},
$$

rendering Lie color algebras as another instance of (2.4). For Lie color algebras the assumption $\chi\left(g_{2}, g_{1}\right)=\chi\left(g_{1}, g_{2}\right)^{-1}$ for all $g_{1}, g_{2} \in G$ leads to a totally defined multiplication $[.,]:. A \times A \longrightarrow A$ since $\left[x_{1}, x_{2}\right]$ is defined for any pair $x_{1}, x_{2} \in A$ of homogeneous elements. A special new operation is the following bracket product. If $g \in G$ satisfies 
$\chi(g, g)=\zeta \neq 1$ then we have the product $[\ldots]: \otimes^{n} A_{g} \longrightarrow A$

$$
\left[x_{1}, \ldots, x_{n}\right]=\sum_{\sigma \in S_{n}} x_{\sigma(1)} \ldots x_{\sigma(n)} .
$$

Theorem 2.5 ((Anti-)Symmetry). Let $\zeta \in k^{*}$ and $\left(g_{1}, \ldots, g_{n}\right)$ be a $\zeta$-family. Let $x_{i} \in A_{g_{i}}$ and $\sigma \in S_{n}$. Then

$$
\left[x_{1}, \ldots, x_{n}\right]=\rho\left(\sigma,\left(g_{1}, \ldots, g_{n}\right)\right)\left[x_{\sigma(1)}, \ldots, x_{\sigma(n)}\right] .
$$

Pro of. We have

$$
\begin{aligned}
\rho\left(\sigma,\left(g_{1}, \ldots, g_{n}\right)\right) & {\left[x_{\sigma(1)}, \ldots, x_{\sigma(n)}\right]=} \\
& =\sum_{\tau \in S_{n}} \rho\left(\sigma,\left(g_{1}, \ldots, g_{n}\right)\right) \rho\left(\tau,\left(g_{\sigma(1)}, \ldots, g_{\sigma(n)}\right)\right) x_{\sigma \tau(1)} \cdot \ldots \cdot x_{\sigma \tau(n)} \\
& =\sum_{\tau \in S_{n}} \rho\left(\sigma \tau,\left(g_{1}, \ldots, g_{n}\right)\right) x_{\sigma \tau(1)} \cdot \ldots \cdot x_{\sigma \tau(n)} .
\end{aligned}
$$

In the case of Lie algebras this amounts to

$$
\left[x_{1}, x_{2}\right]=-\left[x_{2}, x_{1}\right]
$$

in the case of Lie super algebras this is

$$
\left[x_{1}, x_{2}\right]=\left[x_{2}, x_{1}\right]
$$

(for $\zeta=-1, n=2$ and $\chi\left(g_{1}, g_{2}\right)=\chi\left(g_{2}, g_{1}\right)=-1$ ); and in the case of Lie color algebras (see $[\mathrm{FM}] 3.11$ ) this is

$$
\left[x_{1}, x_{2}\right]=-\chi\left(g_{1}, g_{2}\right)\left[x_{2}, x_{1}\right] .
$$

In the following theorem let $(i \ldots 1)=\left(\begin{array}{c}1,2, \ldots, i, i+1, \ldots, n+1 \\ i, 1, \ldots, i-1, i+1, \ldots, n+1\end{array}\right)$ denote a cycle in $S_{n+1}$.

THEOREM 2.6 (Jacobi identities).

1. Let $\left(g_{1}, \ldots, g_{n+1}\right)$ be a $\zeta$-family with $\zeta$ a primitive $n$-th root of unity. Then

$$
\sum_{i=1}^{n+1} \rho\left((i \ldots 1),\left(g_{1}, \ldots, g_{n+1}\right)\right)\left[x_{i},\left[x_{1}, \ldots, \hat{x}_{i}, \ldots, x_{n+1}\right]\right]=0
$$

for all $x_{i} \in A_{g_{i}}$.

2. Let $\left(g_{1}, \ldots, g_{n}\right)$ be a $\zeta$-family with $\zeta$ a primitive $n$-th root of unity and let $h \in G$ such that all $\left(h, g_{i}\right)$ are $(-1)$-families. Then

$$
\left[x,\left[y_{1}, y_{2}, \ldots, y_{n}\right]\right]=\sum_{i=1}^{n}\left(\prod_{j=1}^{i-1} \chi\left(g_{j}, h\right)\right)\left[y_{1}, \ldots,\left[x, y_{i}\right], \ldots, y_{n}\right]
$$

for all $x \in A_{h}$ and $y_{i} \in A_{g_{i}}$.

Proof. (1) Let $\tau \in S_{n}$. Construct $\bar{\tau} \in S_{n+1}$ by $\bar{\tau}(1)=1$ and $\bar{\tau}(j)=\tau(j-1)+1$. This defines a bijection between $S_{n}$ and the set of all $\sigma \in S_{n+1}$ with $\sigma(1)=1$. Using $h_{i}:=g_{i+1}$ we get

$$
\begin{aligned}
\rho\left(\tau,\left(g_{2}, \ldots, g_{n+1}\right)\right) & =\rho\left(\tau,\left(h_{1}, \ldots, h_{n}\right)\right) \\
& =\prod_{1 \leq i<j \leq n, \tau(i)>\tau(j)}\left(\zeta^{-1} \chi\left(h_{\tau(j)}, h_{\tau(i)}\right)\right)
\end{aligned}
$$




$$
\begin{aligned}
& =\prod_{2 \leq i<j \leq n+1, \tau(i-1)>\tau(j-1)}\left(\zeta^{-1} \chi\left(g_{\tau(j-1)+1}, g_{\tau(i-1)+1}\right)\right) \\
& =\prod_{1 \leq i<j \leq n+1, \bar{\tau}(i)>\bar{\tau}(j)}\left(\zeta^{-1} \chi\left(g_{\bar{\tau}(j)}, g_{\bar{\tau}(i)}\right)\right) \\
& =\rho\left(\bar{\tau},\left(g_{1}, \ldots, g_{n+1}\right)\right)
\end{aligned}
$$

So we get for all $i \in\{1, \ldots, n+1\}$

$$
\rho\left(\tau,\left(g_{1}, \ldots, g_{i-1}, \hat{g}_{i}, g_{i+1}, \ldots, g_{n+1}\right)\right)=\rho\left(\bar{\tau},\left(g_{i}, g_{1}, \ldots, g_{i-1}, \hat{g}_{i}, g_{i+1}, \ldots, g_{n+1}\right)\right) .
$$

Now let $\sigma=\sigma_{i} \in S_{n+1}$ with $i:=\sigma(1)$. Let $\bar{\tau}:=(1 \ldots i) \sigma_{i}$. Then $\bar{\tau}(1)=1$, so $\bar{\tau}$ comes from some $\tau \in S_{n}$. Furthermore $\sigma_{i}=(i \ldots 1) \bar{\tau}$. By Lemma 2.2 we get

$$
\begin{aligned}
\rho\left(\sigma_{i},\left(g_{1}, \ldots, g_{n+1}\right)\right) & =\rho\left(\bar{\tau},\left(g_{i}, g_{1}, \ldots, \hat{g}_{i}, \ldots, g_{n+1}\right)\right) \rho\left((i \ldots 1),\left(g_{1}, \ldots, g_{n+1}\right)\right) \\
& =\rho\left(\tau,\left(g_{1}, \ldots, \hat{g}_{i}, \ldots, g_{n+1}\right)\right) \rho\left((i \ldots 1),\left(g_{1}, \ldots, g_{n+1}\right)\right) .
\end{aligned}
$$

Given $\tau \in S_{n}$ we define $\tilde{\tau}$ by $\tilde{\tau}(n+1):=n+1$ and $\tilde{\tau}(j):=\tau(j)$ else. This defines a bijection between $S_{n}$ and the set of all $\sigma \in S_{n+1}$ with $\sigma(n+1)=n+1$. Then we get

$$
\rho\left(\tau,\left(g_{1}, \ldots, g_{n}\right)\right)=\rho\left(\tilde{\tau},\left(g_{1}, \ldots, g_{n+1}\right)\right)
$$

and

$$
\rho\left(\tau,\left(g_{1}, \ldots, \hat{g}_{i}, \ldots, g_{n+1}\right)\right)=\rho\left(\tilde{\tau},\left(g_{1}, \ldots, \hat{g}_{i}, \ldots, g_{n+1}, g_{i}\right)\right) .
$$

Now let $\sigma=\sigma^{i} \in S_{n+1}$ with $i:=\sigma(n+1)$. Let $\tilde{\tau}:=(n+1 \ldots i) \sigma^{i}$. Then $\tilde{\tau}(n+1)=n+1$, so $\tilde{\tau}$ comes from some $\tau \in S_{n}$. Furthermore $\sigma^{i}=(i \ldots n+1) \tilde{\tau}$ and we get

$$
\rho\left(\sigma^{i},\left(g_{1}, \ldots, g_{n+1}\right)\right)=\rho\left(\tau,\left(g_{1}, \ldots, \hat{g}_{i}, \ldots, g_{n+1}\right)\right) \rho\left((i \ldots n+1),\left(g_{1}, \ldots, g_{n+1}\right)\right) .
$$

To prove the Jacobi identity we observe that since $\zeta$ is a primitive $n$-th root of unity

$$
\begin{aligned}
\chi\left(g_{i}, g_{1}+\ldots+\hat{g}_{i}+\ldots+g_{n+1}\right) \chi & \left(g_{1}+\ldots+\hat{g}_{i}+\ldots+g_{n+1}, g_{i}\right) \\
& =\prod_{j \neq i} \chi\left(g_{i}, g_{j}\right) \chi\left(g_{j}, g_{i}\right)=\zeta^{2 n}=1,
\end{aligned}
$$

hence we have a $(-1)$-family $\left(g_{i}, g_{1}+\ldots+\hat{g}_{i}+\ldots+g_{n+1}\right)$ and

$$
\begin{aligned}
& {\left[x_{i},\left[x_{1}, \ldots, \hat{x}_{i}, \ldots, x_{n+1}\right]\right]=} \\
& \quad=x_{i}\left[x_{1}, \ldots, \hat{x}_{i}, \ldots, x_{n+1}\right]-\left(\prod_{j \neq i} \chi\left(g_{i}, g_{j}\right)\right)\left[x_{1}, \ldots, \hat{x}_{i}, \ldots, x_{n+1}\right] x_{i} .
\end{aligned}
$$

Furthermore we use $\zeta^{n}=1$ to get

$$
\begin{aligned}
\rho\left((i \ldots 1),\left(g_{1}, \ldots, g_{n+1}\right)\right) & \prod_{j \neq i} \chi\left(g_{i}, g_{j}\right)= \\
& =\left(\prod_{j<i}\left(\zeta^{-1} \chi\left(g_{j}, g_{i}\right)\right)\right)\left(\prod_{j \neq i}\left(\zeta^{-1} \chi\left(g_{i}, g_{j}\right)\right)\right) \\
& =\prod_{j>i}\left(\zeta^{-1} \chi\left(g_{i}, g_{j}\right)\right)=\rho\left((i \ldots n+1),\left(g_{1}, \ldots, g_{n+1}\right)\right) .
\end{aligned}
$$

With these notations we can now evaluate

$$
\sum_{i=1}^{n+1} \rho\left((i \ldots 1),\left(g_{1}, \ldots, g_{n+1}\right)\right)\left[x_{i},\left[x_{1}, \ldots, \hat{x}_{i}, \ldots, x_{n+1}\right]\right]=
$$




$$
\begin{aligned}
& =\sum_{i=1}^{n+1} \rho\left((i \ldots 1),\left(g_{1}, \ldots, g_{n+1}\right)\right) x_{i}\left[x_{1}, \ldots, \hat{x}_{i}, \ldots, x_{n+1}\right] \\
& -\rho\left((i \ldots 1),\left(g_{1}, \ldots, g_{n+1}\right)\right)\left(\prod_{j \neq i} \chi\left(g_{i}, g_{j}\right)\right)\left[x_{1}, \ldots, \hat{x}_{i}, \ldots, x_{n+1}\right] x_{i} \\
= & \sum_{i=1}^{n+1} \sum_{\tau \in S_{n}} \rho\left((i \ldots 1),\left(g_{1}, \ldots, g_{n+1}\right)\right) \rho\left(\tau,\left(g_{1}, \ldots, \hat{g}_{i}, \ldots, g_{n+1}\right)\right) x_{\sigma_{i}(1)} \ldots x_{\sigma_{i}(n+1)} \\
& -\rho\left((i \ldots n+1),\left(g_{1}, \ldots, g_{n+1}\right)\right) \rho\left(\tau,\left(g_{1}, \ldots, \hat{g}_{i}, \ldots, g_{n+1}\right)\right) x_{\sigma^{i}(1)} \ldots x_{\sigma^{i}(n+1)} \\
= & \sum_{\sigma \in S_{n+1}} \rho\left(\sigma,\left(g_{1}, \ldots, g_{n+1}\right)\right) x_{\sigma(1)} \ldots x_{\sigma(n+1)}-\rho\left(\sigma,\left(g_{1}, \ldots, g_{n+1}\right)\right) x_{\sigma(1)} \ldots x_{\sigma(n+1)} \\
= & 0
\end{aligned}
$$

(2) Since $\chi\left(h, g_{i}\right) \chi\left(g_{i}, h\right)=(-1)^{2}$ and $\chi\left(g_{i}, g_{j}\right) \chi\left(g_{j}, g_{i}\right)=\zeta^{2}$ we have $\chi\left(h, g_{1}+\ldots+\right.$ $\left.g_{n}\right) \chi\left(g_{1}+\ldots+g_{n}, h\right)=1$ and $\chi\left(g_{i}, h+g_{j}\right) \chi\left(h+g_{j}, g_{i}\right)=\zeta^{2}$ so that all terms are defined.

Let $\sigma \in S_{n}$ with $\sigma(j)=i$. Then we have

$$
\begin{aligned}
& \rho\left(\sigma,\left(g_{1}, \ldots, g_{i-1}, h+g_{i}, g_{i+1}, \ldots, g_{n}\right)\right)= \\
& \quad=\left(\prod_{k<l, \sigma(k)>\sigma(l)}\left(\zeta^{-1} \chi\left(g_{\sigma(l)}, g_{\sigma(k)}\right)\right)\right)\left(\prod_{j<l, i>\sigma(l)} \chi\left(g_{\sigma(l)}, h\right)\right)\left(\prod_{k<j, \sigma(k)>i} \chi\left(h, g_{\sigma(k)}\right)\right) \\
& \quad=\rho\left(\sigma,\left(g_{1}, \ldots, g_{n}\right)\right)\left(\prod_{\sigma^{-1}(i)<l, i>\sigma(l)} \chi\left(g_{\sigma(l)}, h\right)\right)\left(\prod_{k<\sigma^{-1}(i), \sigma(k)>i} \chi\left(h, g_{\sigma(k)}\right)\right) .
\end{aligned}
$$

We abbreviate $z_{k}:=u_{k}:=y_{k}$ for $k \neq i, z_{i}:=x y_{i}$, and $u_{i}:=y_{i} x$. Then we get

$$
\begin{aligned}
\sum_{i=1}^{n} & \left(\prod_{r=1}^{i-1} \chi\left(h, g_{r}\right)\right)\left[y_{1}, \ldots,\left[x, y_{i}\right], \ldots, y_{n}\right] \\
= & \sum_{i=1}^{n}\left(\prod_{r=1}^{i-1} \chi\left(h, g_{r}\right)\right)\left[y_{1}, \ldots, x y_{i}-\chi\left(h, g_{i}\right) y_{i} x, \ldots, y_{n}\right] \\
= & \sum_{i=1}^{n} \sum_{\sigma \in S_{n}}\left(\left(\prod_{r=1}^{i-1} \chi\left(h, g_{r}\right)\right) \rho\left(\sigma,\left(g_{1}, \ldots, h+g_{i}, \ldots, g_{n}\right)\right) z_{\sigma(1)} \ldots z_{\sigma(n)}\right. \\
& \left.-\left(\prod_{r=1}^{i-1} \chi\left(h, g_{r}\right)\right) \chi\left(h, g_{i}\right) \rho\left(\sigma,\left(g_{1}, \ldots, h+g_{i}, \ldots, g_{n}\right)\right) u_{\sigma(1)} \ldots u_{\sigma(n)}\right) \\
= & \sum_{\sigma \in S_{n}} \sum_{j=1}^{n}\left(\prod_{r=1}^{\sigma(j)-1} \chi\left(h, g_{r}\right)\right) \rho\left(\sigma,\left(g_{1}, \ldots, g_{n}\right)\right)\left(\prod_{j<l, \sigma(j)>\sigma(l)} \chi\left(g_{\sigma(l)}, h\right)\right) . \\
& \cdot\left(\prod_{l<j, \sigma(l)>\sigma(j)} \chi\left(h, g_{\sigma(l)}\right)\right) y_{\sigma(1)} \ldots x y_{\sigma(j)} \ldots y_{\sigma(n)} \\
& -\sum_{\sigma \in S_{n}} \sum_{j=1}^{n}\left(\prod_{r=1}^{\sigma(j)-1} \chi\left(h, g_{r}\right)\right) \chi\left(h, g_{\sigma(j)}\right) \rho\left(\sigma,\left(g_{1}, \ldots, g_{n}\right)\right)\left(\prod_{j<l, \sigma(j)>\sigma(l)} \chi\left(g_{\sigma(l)}, h\right)\right) . \\
& \cdot\left(\prod_{l<j, \sigma(l)>\sigma(j)} \chi\left(h, g_{\sigma(l)}\right)\right) y_{\sigma(1)} \ldots y_{\sigma(j)} x \ldots y_{\sigma(n)}
\end{aligned}
$$




$$
\begin{aligned}
& =\sum_{\sigma \in S_{n}} \rho\left(\sigma,\left(g_{1}, \ldots, g_{n}\right)\right) x y_{\sigma(1)} \ldots y_{\sigma(n)} \\
& -\sum_{\sigma \in S_{n}}\left(\prod_{r=1}^{n} \chi\left(h, g_{r}\right)\right) \rho\left(\sigma,\left(g_{1}, \ldots, g_{n}\right)\right) y_{\sigma(1)} \ldots y_{\sigma(n)} x \\
= & {\left[x,\left[y_{1}, \ldots, y_{n}\right]\right] . }
\end{aligned}
$$

if we can show that the coefficients reduce appropriately. For the first term $(j=1)$ of the first sum and the last term $(j=n)$ of the second sum this is easy to see. The other terms cancel for all $\sigma \in S_{n}$ and all $j$. In fact, let $q:=\sigma(j)$ and $p=\sigma(j-1)$. We have to show

$$
\begin{gathered}
\left(\prod_{r=1}^{\sigma(j)-1} \chi\left(h, g_{r}\right)\right)\left(\prod_{j<l, \sigma(j)>\sigma(l)} \chi\left(g_{\sigma(l)}, h\right)\right)\left(\prod_{l<j, \sigma(l)>\sigma(j)} \chi\left(h, g_{\sigma(l)}\right)\right) \\
=\left(\prod_{r=1}^{\sigma(j-1)-1} \chi\left(h, g_{r}\right)\right) \chi\left(h, g_{\sigma(j-1)}\right)\left(\prod_{j-1<l, \sigma(j-1)>\sigma(l)} \chi\left(g_{\sigma(l)}, h\right)\right) . \\
\cdot\left(\prod_{l<j-1, \sigma(l)>\sigma(j-1)} \chi\left(h, g_{\sigma(l)}\right)\right)
\end{gathered}
$$

We change parameters by $\tau=\sigma^{-1}$ with $\tau(p)+1=\tau(q)$ and have to show

$$
\begin{aligned}
& \left(\prod_{r=1}^{q-1} \chi\left(h, g_{r}\right)\right)\left(\prod_{q>l, \tau(q)<\tau(l)} \chi\left(g_{l}, h\right)\right)\left(\prod_{l>q, \tau(l)<\tau(q)} \chi\left(h, g_{l}\right)\right) \\
& \quad=\left(\prod_{r=1}^{p} \chi\left(h, g_{r}\right)\right)\left(\prod_{p>l, \tau(p)<\tau(l)} \chi\left(g_{l}, h\right)\right)\left(\prod_{l>p, \tau(l)<\tau(p)} \chi\left(h, g_{l}\right)\right) .
\end{aligned}
$$

This can be easily checked if one considers the cases $p<q$ and $p>q$ separately.

3. Primitive elements. We come to the main technical theorem of this paper which has applications to primitive elements in Hopf algebras.

Theorem 3.1. Let $A$ be a $(G, \chi)$-algebra in $\mathcal{M}^{k G}$. Then the following hold in $A \otimes A$

$$
\left[x_{1} \otimes 1+1 \otimes x_{1}, \ldots, x_{n} \otimes 1+1 \otimes x_{n}\right]=\left[x_{1}, \ldots, x_{n}\right] \otimes 1+1 \otimes\left[x_{1}, \ldots, x_{n}\right]
$$

for all primitive $n$-th roots of unity $\zeta$, all $\zeta$-families $\left(g_{1}, \ldots, g_{n}\right)$, and all $x_{i} \in A_{g_{i}}$.

Proof. We have to evaluate

$$
\begin{aligned}
{\left[x_{1} \otimes 1\right.} & \left.+1 \otimes x_{1}, \ldots, x_{n} \otimes 1+1 \otimes x_{n}\right] \\
& =\sum_{\sigma \in S_{n}} \rho\left(\sigma,\left(g_{1}, \ldots, g_{n}\right)\right)\left(x_{\sigma(1)} \otimes 1+1 \otimes x_{\sigma(1)}\right) \ldots\left(x_{\sigma(n)} \otimes 1+1 \otimes x_{\sigma(n)}\right) .
\end{aligned}
$$

Observe that

$$
\begin{aligned}
& \left(x_{i} \otimes 1\right)\left(x_{j} \otimes 1\right)=\left(x_{i} x_{j} \otimes 1\right), \\
& \left(x_{i} \otimes 1\right)\left(1 \otimes x_{j}\right)=\left(x_{i} \otimes x_{j}\right), \\
& \left(1 \otimes x_{i}\right)\left(1 \otimes x_{j}\right)=\left(1 \otimes x_{i} x_{j}\right), \\
& \left(1 \otimes x_{i}\right)\left(x_{j} \otimes 1\right)=\chi\left(g_{i}, g_{j}\right)\left(x_{j} \otimes x_{i}\right) .
\end{aligned}
$$


We collect all terms of the form $c_{\sigma, i} \cdot x_{\sigma(1)} \ldots x_{\sigma(i)} \otimes x_{\sigma(i+1)} \ldots x_{\sigma(n)}$ with $\sigma \in S_{n}$ and want to show that they are zero for all $1<i<n$.

By Theorem $2.5\left[x_{1} \otimes 1+1 \otimes x_{1}, \ldots, x_{n} \otimes 1+1 \otimes x_{n}\right]$ and $\left[x_{\sigma(1)} \otimes 1+1 \otimes x_{\sigma(1)}, \ldots, x_{\sigma(n)} \otimes\right.$ $\left.1+1 \otimes x_{\sigma(n)}\right]$ have (generically) proportional terms, in particular $c_{\sigma, i} \cdot x_{\sigma(1)} \ldots x_{\sigma(i)} \otimes$ $x_{\sigma(i+1)} \ldots x_{\sigma(n)}=\rho\left(\sigma,\left(g_{1}, \ldots, g_{n}\right)\right) c_{1, i} \cdot x_{\sigma(1)} \ldots x_{\sigma(i)} \otimes x_{\sigma(i+1)} \ldots x_{\sigma(n)}$. So we may just consider the case $\sigma=1$ or the term $c_{1, i} \cdot x_{1} \ldots x_{i} \otimes x_{i+1} \ldots x_{n}$.

The term $c_{1, n} \cdot x_{1} \ldots x_{n} \otimes 1$ occurs only in the product $\left(x_{1} \otimes 1+1 \otimes x_{1}\right)\left(x_{2} \otimes 1+\right.$ $\left.1 \otimes x_{2}\right) \ldots\left(x_{n} \otimes 1+1 \otimes x_{n}\right)$ with the factor $c_{1, n}=1$. The same holds for the term $c_{1,1} \cdot 1 \otimes x_{1} \ldots x_{n}$. Now we consider exclusively terms $c_{1, i} \cdot x_{1} \ldots x_{i} \otimes x_{i+1} \ldots x_{n}$ with $0<i<n$.

We study which terms of the expansion of

$$
\sum_{\sigma \in S_{n}} \rho\left(\sigma,\left(g_{1}, \ldots, g_{n}\right)\right)\left(x_{\sigma(1)} \otimes 1+1 \otimes x_{\sigma(1)}\right) \ldots\left(x_{\sigma(n)} \otimes 1+1 \otimes x_{\sigma(n)}\right)
$$

contribute to $c_{1, i} \cdot x_{1} \ldots x_{i} \otimes x_{i+1} \ldots x_{n}$. This will be those products of factors $x_{1} \otimes$ $1, \ldots, x_{i} \otimes 1$ and $1 \otimes x_{i+1}, \ldots, 1 \otimes x_{n}$ where the terms $x_{1} \otimes 1, \ldots, x_{i} \otimes 1$ occur in the given natural order possibly interrupted by factors from the second set and similarly the terms $1 \otimes x_{i+1}, \ldots, 1 \otimes x_{n}$ occur in the given natural order possibly interrupted by factors from the first set. Such a product will occur in the expansion of

$$
\rho\left(\sigma,\left(g_{1}, \ldots, g_{n}\right)\right)\left(x_{\sigma(1)} \otimes 1+1 \otimes x_{\sigma(1)}\right) \ldots\left(x_{\sigma(n)} \otimes 1+1 \otimes x_{\sigma(n)}\right)
$$

whenever $\sigma \in S_{n}$ is a shuffle of $\{1, \ldots, i\}$ with $\{i+1, \ldots, n\}$, i.e. if $1 \leq j<k \leq i$ or $i+1 \leq j<k \leq n$ then $\sigma^{-1}(j)<\sigma^{-1}(k)$.

To evaluate such a product we have to interchange factors according to the rule given above, namely,

$\left(1 \otimes x_{m}\right)\left(x_{l} \otimes 1\right)=\chi\left(g_{m}, g_{l}\right)\left(x_{l} \otimes x_{m}\right)=\chi\left(g_{m}, g_{l}\right)\left(x_{l} \otimes 1\right)\left(1 \otimes x_{m}\right)$ for $1 \leq l \leq i<m \leq n$.

This rule has to be applied for every pair in the product (and in $\sigma$ ) in reverse position $j<k$ and $m:=\sigma(j)>\sigma(k)=l$ thus producing a factor $\chi\left(g_{\sigma(j)}, g_{\sigma(k)}\right)$. So the total contribution of

$$
\begin{aligned}
& \rho\left(\sigma,\left(g_{1}, \ldots, g_{n}\right)\right)\left(x_{\sigma(1)} \otimes 1+1 \otimes x_{\sigma(1)}\right) \ldots\left(x_{\sigma(n)} \otimes 1+1 \otimes x_{\sigma(n)}\right) \\
& \text { to } c_{1, i} \cdot x_{1} \ldots x_{i} \otimes x_{i+1} \ldots x_{n} \text { is } \\
& \rho\left(\sigma,\left(g_{1}, \ldots, g_{n}\right)\right) \\
& \quad \prod_{j<k, \sigma(j)>\sigma(k)} \chi\left(g_{\sigma(j)}, g_{\sigma(k)}\right) \\
& \left.=\prod_{j<k, \sigma(j)>\sigma(k)}\left(\zeta^{-1} \chi\left(g_{\sigma(k)}, g_{\sigma(j)}\right)\right) \chi\left(g_{\sigma(j)}, g_{\sigma(k)}\right)\right)=\zeta^{t}
\end{aligned}
$$

where $t$ is the number of pairs in reverse position in $\sigma$. Here we have used that $\left(g_{1}, \ldots, g_{n}\right)$ is a $\zeta$-family.

To determine the number $t$ of pairs in reverse position for a fixed $\sigma$ we observe that the ordering of $1, \ldots, i$ and of $i+1, \ldots, n$ must be preserved in the product. We count how many steps the factor $1 \otimes x_{i+1}$ has been moved to the left or how many terms from $x_{1} \otimes 1, \ldots, x_{i} \otimes 1$ in the product are to the right of $1 \otimes x_{i+1}$ and call this number $\lambda_{1}$. Observe that $0 \leq \lambda_{1} \leq i$. Similarly $\lambda_{2}$ denotes the number of terms from $x_{1} \otimes 1, \ldots, x_{i} \otimes 1$ 
in the product that are to the right of $1 \otimes x_{i+2}$. We have $0 \leq \lambda_{2} \leq \lambda_{1}$. In a similar way we continue to define the numbers $\lambda_{j}$ with $0 \leq \lambda_{n-i} \leq \ldots \leq \lambda_{2} \leq \lambda_{1} \leq i$. The evaluation of the selected product then gives a term

$$
\zeta^{\lambda_{1}+\ldots+\lambda_{n-i}} x_{1} \ldots x_{i} \otimes x_{i+1} \ldots x_{n} .
$$

To get the number of terms $\zeta^{t} x_{1} \ldots x_{i} \otimes x_{i+1} \ldots x_{n}$ in $\left[x_{1} \otimes 1+1 \otimes x_{1}, \ldots, x_{n} \otimes 1+1 \otimes x_{n}\right]$ we have to count all possibilities to represent $t=\lambda_{1}+\ldots+\lambda_{n-i}$ with $0 \leq \lambda_{n-i} \leq \ldots \leq$ $\lambda_{2} \leq \lambda_{1} \leq i$ or the number $p(i, n-i, t)$ of partitions of $t$ into at most $n-i$ parts each $\leq i$. Thus we can now determine the factor $c_{1, i}$ for $c_{1, i} \cdot x_{1} \ldots x_{i} \otimes x_{i+1} \ldots x_{n}$ in the expansion of $\left[x_{1} \otimes 1+1 \otimes x_{1}, \ldots, x_{n} \otimes 1+1 \otimes x_{n}\right]$ as

$$
c_{1, i}=\sum_{t \geq 0} p(i, n-i, t) \zeta^{t} .
$$

By a theorem of Sylvester ([A] Theorem 3.1) we have

$$
\sum_{t \geq 0} p(i, n-i, t) q^{t}=\frac{\left(1-q^{n}\right)\left(1-q^{n-1}\right) \ldots\left(1-q^{n-i+1}\right)}{\left(1-q^{i}\right)\left(1-q^{i-1}\right) \ldots(1-q)}
$$

hence $c_{1, i}=0$ for $1<i<n$ since $\zeta$ is a primitive $n$-th root of unity (see also [T] p. 2632).

So we have shown

$$
\begin{aligned}
{\left[x_{1} \otimes 1\right.} & \left.+1 \otimes x_{1}, \ldots, x_{n} \otimes 1+1 \otimes x_{n}\right]= \\
& =\sum_{\sigma \in S_{n}} \rho\left(\sigma,\left(g_{1}, \ldots, g_{n}\right)\right) x_{\sigma(1)} \ldots x_{\sigma(n)} \otimes 1+1 \otimes x_{\sigma(1)} \ldots x_{\sigma(n)} \\
& =\left[x_{1}, \ldots, x_{n}\right] \otimes 1+1 \otimes\left[x_{1}, \ldots, x_{n}\right] .
\end{aligned}
$$

Now we will study Hopf algebras in the category $\mathcal{M}^{k G}$ and their primitive $\left.{ }^{1}\right)$ elements. An element $x \in H$ is primitive if $\Delta(x)=x \otimes 1+1 \otimes x$. The set of primitive elements of an ordinary Hopf algebra forms a Lie algebra. This is not true for Hopf algebras in $\mathcal{M}^{k G}$.

Let $P(H)$ denote the set of primitive elements of $H$ and $P_{g}(H)$ the set of primitive elements of degree $g$ in $H$. Then

$$
P(H)=\bigoplus_{g \in G} P_{g}(H)
$$

since for any $x=\sum_{g \in G} x_{g}$ we have

$$
\sum_{g \in G} \Delta\left(x_{g}\right)=\Delta(x)=x \otimes 1+1 \otimes x=\left(\sum_{g \in G} x_{g}\right) \otimes 1+1 \otimes\left(\sum_{g \in G} x_{g}\right),
$$

so by comparing homogeneous terms we get $\Delta\left(x_{g}\right)=x_{g} \otimes 1+1 \otimes x_{g}$. Thus the homogeneous components $x_{g}$ of $x$ are again primitive hence in $P_{g}(H)$ which shows $P(H) \subseteq$ $\bigoplus_{g \in G} P_{g}(H)$. The converse inclusion is trivial.

TheOREm 3.2. Let $H$ be a $(G, \chi)$-Hopf algebra in $\mathcal{M}^{k G}$. Then for all primitive $n$-th roots of unity $\zeta \neq 1$ and all $\zeta$-families $\left(g_{1}, \ldots, g_{n}\right)$ the following is a linear map

$$
[\ldots]: P_{g_{1}}(H) \otimes \ldots \otimes P_{g_{n}}(H) \longrightarrow P_{g_{1}+\ldots+g_{n}}(H)
$$

$\left({ }^{1}\right)$ This notion of primitivity is not related to the notion of primitivity for roots of unity. 


$$
\left[x_{1}, \ldots, x_{n}\right]:=\sum_{\sigma \in S_{n}} \rho\left(\sigma,\left(g_{1}, \ldots, g_{n}\right)\right) x_{\sigma(1)} \ldots x_{\sigma(n)} .
$$

Proof. It is clear that the degree of each product $x_{\sigma(1)} \ldots x_{\sigma(n)}$ is $g_{1}+\ldots+g_{n}$. So we only have to show that $\left[x_{1}, \ldots, x_{n}\right]$ is primitive. But that is a consequence of Theorem 3.1 .

Observe that we have special multiplications

$$
[\ldots]: P_{g}(H) \otimes \ldots \otimes P_{g}(H) \longrightarrow P_{n g}(H)
$$

for all $g \in G$ with $|g| \neq 1$ a primitive $n$-th root of unity.

Definition 3.3. Let $A$ be an algebra in $\mathcal{M}^{k G}$. A derivation from $A$ to $A$ of degree $g \in G$ is a family of linear maps $\left(d_{h}: A_{h} \longrightarrow A_{h+g} \mid h \in G\right)$ such that

$$
d(a b)=d(a) b+\chi(g, h) a d(b)
$$

for all $a \in A_{h}, b \in A_{h^{\prime}}$, all $h, h^{\prime} \in G$.

It is clear that all derivations from $A$ to $A$ of all degrees form an object $\operatorname{Der}(A)$ in $\mathcal{M}^{k G}$ and that there is an operation $\operatorname{Der}(A) \otimes A \longrightarrow A$.

Corollary 3.4. $\operatorname{Der}(A)$ is a $(G, \chi)$-Lie algebra.

Proof. Let $m$ denote the multiplication of $A$. An endomorphism $x: A \longrightarrow A$ of degree $g \in G$, i.e. $x_{h}: A_{h} \longrightarrow A_{h+g}$ for all $h \in G$ is a derivation iff $m(x \otimes 1+1 \otimes x)=x m$ where $(x \otimes y)(a \otimes b)=\chi(\operatorname{deg}(y), \operatorname{deg}(a)) x(a) \otimes y(b)$ for homogeneous elements $a$ and $b$ in A.

To show that $\operatorname{Der}(A)$ is a Lie algebra it suffices to show that it is closed under Lie multiplication since it is a subobject of $\operatorname{End}(A)$, the inner endomorphism object of $A$ which is known to be an algebra in the category $\mathcal{M}^{k G}$. Let $\zeta$ be a primitive $n$-th root of unity and let $\left(g_{1}, \ldots, g_{n}\right)$ be a $\zeta$-family. Then

$$
\begin{aligned}
m( & {\left.\left[x_{1}, \ldots, x_{n}\right] \otimes 1+1 \otimes\left[x_{1}, \ldots, x_{n}\right]\right) } \\
& =m\left[x_{1} \otimes 1+1 \otimes x_{1}, \ldots, x_{n} \otimes 1+1 \otimes x_{n}\right] \\
& =m \sum_{\sigma \in S_{n}} \rho\left(\sigma,\left(g_{1}, \ldots, g_{n}\right)\right)\left(x_{\sigma(1)} \otimes 1+1 \otimes x_{\sigma(1)}\right) \cdot \ldots \cdot\left(x_{\sigma(n)} \otimes 1+1 \otimes x_{\sigma(n)}\right) \\
& =\sum_{\sigma \in S_{n}} \rho\left(\sigma,\left(g_{1}, \ldots, g_{n}\right)\right) m\left(x_{\sigma(1)} \otimes 1+1 \otimes x_{\sigma(1)}\right) \cdot \ldots \cdot\left(x_{\sigma(n)} \otimes 1+1 \otimes x_{\sigma(n)}\right) \\
& =\sum_{\sigma \in S_{n}} \rho\left(\sigma,\left(g_{1}, \ldots, g_{n}\right)\right) x_{\sigma(1)} \cdot \ldots \cdot x_{\sigma(n)} \cdot m \\
& =\left[x_{1}, \ldots, x_{n}\right] m
\end{aligned}
$$

for all derivations $x_{1}, \ldots, x_{n}$ of degrees $g_{1}, \ldots, g_{n}$ respectively. Hence $\left[x_{1}, \ldots, x_{n}\right]$ again is a derivation.

\section{Lie algebras and universal enveloping algebras}

Definition 4.1. An object $P=\bigoplus P_{g} \in \mathcal{M}^{k G}$ together with operations

$$
[\ldots]: P_{g_{1}} \otimes \ldots \otimes P_{g_{n}} \longrightarrow P_{g_{1}+\ldots+g_{n}}
$$


for all $n \in \mathbf{N}$, all primitive $n$-th roots of unity $\zeta$, and all $\zeta$-families $\left(g_{1}, \ldots, g_{n}\right)$, is called a $(G, \chi)$-Lie algebra if the following identities hold:

1. for all primitive $n$-th roots of unity $\zeta$, all $\zeta$-families $\left(g_{1}, \ldots, g_{n}\right)$, all $\sigma \in S_{n}$, and all $x_{i} \in P_{g_{i}}$

$$
\left[x_{1}, \ldots, x_{n}\right]=\rho\left(\sigma,\left(g_{1}, \ldots, g_{n}\right)\right)\left[x_{\sigma(1)}, \ldots, x_{\sigma(n)}\right],
$$

2. for all primitive $n$-th roots of unity $\zeta$, all $\zeta$-families $\left(g_{1}, \ldots, g_{n+1}\right)$, and for all $x_{i} \in P_{g_{i}}$

$$
\sum_{i=1}^{n+1} \rho\left((i \ldots 1),\left(g_{1}, \ldots, g_{n+1}\right)\right)\left[x_{i},\left[x_{1}, \ldots, \hat{x}_{i}, \ldots, x_{n+1}\right]\right]=0,
$$

3. for all primitive $n$-th roots of unity $\zeta$, all $\zeta$-families $\left(g_{1}, \ldots, g_{n}\right)$, all $h \in G$ such that all $\left(h, g_{i}\right)$ are $(-1)$-families, all $y_{i} \in P_{g_{i}}$, and all $x \in P_{h}$

$$
\left[x,\left[y_{1}, y_{2}, \ldots, y_{n}\right]\right]=\sum_{i=1}^{n}\left(\prod_{j=1}^{i-1} \chi\left(g_{j}, h\right)\right)\left[y_{1}, \ldots,\left[x, y_{i}\right], \ldots, y_{n}\right] .
$$

The $(G, \chi)$-Lie algebras form a category in a straightforward way and the construction given in section 3 defines a functor $P:(G, \chi)$-Hopf $\longrightarrow(G, \chi)$-Lie as can be easily verified.

Theorems 3.2, 2.5, and 2.6 show that this notion of generalized Lie algebras passes its test on the set of primitive elements of a Hopf algebra:

Corollary 4.2. Let $H$ be a $(G, \chi)$-Hopf algebra in $\mathcal{M}^{k G}$. Then the set of primitive elements $P(H)$ forms a $(G, \chi)$-Lie algebra.

Lemma 4.3. Let $A$ be a $(G, \chi)$-algebra in $\mathcal{M}^{k G}$. Then $A$ carries the structure of a $(G, \chi)$-Lie algebra $A^{L}$ with the operations

$$
\left[x_{1}, \ldots, x_{n}\right]:=\sum_{\sigma \in S_{n}} \rho\left(\sigma,\left(g_{1}, \ldots, g_{n}\right)\right) x_{\sigma(1)} \cdot \ldots \cdot x_{\sigma(n)}
$$

for all (roots of unity) $\zeta \in k^{*}$, all $\zeta$-families $\left(g_{1}, \ldots, g_{n}\right)$, and all $x_{i} \in A_{g_{i}}$.

Proof. This is a rephrasing of Theorems 2.5 and 2.6.

This lemma defines a functor $(G, \chi)$ - $\operatorname{Alg} \ni A \mapsto A^{L} \in(G, \chi)$-Lie.

Theorem 4.4. Let $P$ be a $(G, \chi)$-Lie algebra. Then there is a universal associative enveloping algebra $U(P)$ in $\mathcal{M}^{k G}$.

Proof. We define $U(P):=T(P) / I$ where $T(P)$ is the tensor algebra and $I:=$ $\left(\left[x_{1}, \ldots, x_{n}\right]-\sum_{\sigma \in S_{n}} \rho\left(\sigma,\left(g_{1}, \ldots, g_{n}\right)\right) x_{\sigma(1)} \cdot \ldots \cdot x_{\sigma(n)}\right)$ is the ideal generated by all terms formed for all (roots of unity) $\zeta \in k^{*}$, all $\zeta$-families $\left(g_{1}, \ldots, g_{n}\right)$, and all $x_{i} \in P_{g_{i}}$.

The tensor algebra is constructed in $\mathcal{M}^{k G}$ with the natural grading. Since the relations are $G$-homogeneous, the algebra $U(P)$ is also in $\mathcal{M}^{k G}$.

By the universal property of the tensor algebra any $(G, \chi)$-Lie homomorphism $f$ : $P \longrightarrow A^{L}$ extends uniquely to a $(G, \chi)$-algebra homomorphism $g: U(P) \longrightarrow A$, so that $U(P)$ is the universal associative algebra generated by $P$.

Corollary 4.5. The functor ${ }_{-}{ }^{L}:(G, \chi)$-Alg $\longrightarrow(G, \chi)$-Lie has the left adjoint functor $U:(G, \chi)$-Lie $\longrightarrow(G, \chi)$-Alg. 
Observe that the identities for $(G, \chi)$-Lie algebras play no special role in the construction of the universal enveloping algebra. Its construction depends only on the given operations, which must be homogeneous so that the residue class algebra is $G$-graded again.

If we consider the set $P(H)$ of primitive elements of an arbitrary $(G, \chi)$-Hopf algebra $H$ then there are many homogeneous partially defined common operations in all $P(H)$. We used the most basic ones in our generalization of Lie algebras. We would like to know if the bracket-operations and identities given in the definition of a $(G, \chi)$-Lie algebra are a generating set for all possible operations and relations for the $G$-graded module $P(H)$ for every Hopf algebra in $\mathcal{M}^{k G}$, every abelian group $G$, every bicharacter $\chi$ and every integral domain $k$. This should be true for the identities if all maps from $(G, \chi)$-Lie algebras into their universal enveloping algebras are injective. To prove this, some kind of generalization of the Poincaré-Birkhoff-Witt theorem is needed.

Now we show how to construct Hopf algebras in the braided category of $G$-graded vector spaces using universal enveloping algebras..

TheOREm 4.6. Let $P$ be a $(G, \chi)$-Lie algebra. Then the universal enveloping algebra $U(P)$ is a $(G, \chi)$-Hopf algebra.

Proof. We define a homomorphism $\delta: P \longrightarrow(U(P) \otimes U(P))^{L}$ in $\mathcal{M}^{k G}$ by $\delta(x):=$ $\bar{x} \otimes 1+1 \otimes \bar{x}$ where $\bar{x}$ is the canonical image of $x \in P$ in $U(P)$. Then we have

$$
\begin{aligned}
{\left[\delta\left(x_{1}\right), \ldots, \delta\left(x_{n}\right)\right] } & \\
& =\left[\bar{x}_{1} \otimes 1+1 \otimes \bar{x}_{1}, \ldots, \bar{x}_{n} \otimes 1+1 \otimes \bar{x}_{n}\right] \\
& =\left[\bar{x}_{1}, \ldots, \bar{x}_{n}\right] \otimes 1+1 \otimes\left[\bar{x}_{1}, \ldots, \bar{x}_{n}\right] \\
& =\overline{\left[x_{1}, \ldots, x_{n}\right]} \otimes 1+1 \otimes \overline{\left[x_{1}, \ldots, x_{n}\right]} \quad \text { (by Theorem 3.1) } \\
& =\delta\left(\left[x_{1}, \ldots, x_{n}\right]\right) .
\end{aligned}
$$

Hence $\delta: P \longrightarrow(U(P) \otimes U(P))^{L}$ is a $(G, \chi)$-Lie homomorphism that factors uniquely through $\Delta: U(P) \longrightarrow U(P) \otimes U(P)$, an algebra homomorphism. So for all $x \in P$ we have $\Delta(\bar{x})=\bar{x} \otimes 1+1 \otimes \bar{x}$.

Since $(\Delta \otimes 1) \delta(x)=\bar{x} \otimes 1 \otimes 1+1 \otimes \bar{x} \otimes 1+1 \otimes 1 \otimes \bar{x}=(1 \otimes \Delta) \delta(x)$ and since $(\Delta \otimes 1) \delta=(1 \otimes \Delta) \delta$ is a $(G, \chi)$-Lie homomorphism, it factors through a unique algebra homomorphism $(\Delta \otimes 1) \Delta=(1 \otimes \Delta) \Delta$ so that $\Delta: U(P) \longrightarrow U(P) \otimes U(P)$ is coassociative.

The counit $\varepsilon: U(P) \longrightarrow k$ is defined by the zero morphism $0: P \longrightarrow k$. Thus $U(P)$ is a bialgebra.

The definition of the antipode is somewhat more complicated. We consider $U(P)^{o p}$ the opposite algebra of $U(P)$ where the multiplication in $U(P)^{o p}$ is defined by $x_{2} \circ x_{1}=$ $\chi\left(g_{2}, g_{1}\right) x_{1} x_{2}$ for homogeneous elements $x_{i} \in U(P)_{g_{i}}$ hence $x_{1} x_{2}=\chi\left(g_{2}, g_{1}\right)^{-1} x_{2} \circ x_{1}$.

Define a homomorphism $S: P \longrightarrow U(P)^{o p}$ by $S(x):=-\bar{x}$. To show that this is a $(G, \chi)$-Lie homomorphism let $\zeta$ be a primitive $n$-th root of unity, $\left(g_{1}, \ldots, g_{n}\right)$ be a $\zeta$-family, and $x_{i} \in P_{g_{i}}$. We first consider the permutation $\tau \in S_{n}$ with $\tau(i)=n+1-i$. Then $\tau^{2}=1$ and

$$
\rho\left(\tau,\left(g_{1}, \ldots, g_{n}\right)\right)=\prod_{i<j}\left(\zeta^{-1} \chi\left(g_{n+1-j}, g_{n+1-i}\right)\right)=\prod_{i<j}\left(\zeta \chi\left(g_{j}, g_{i}\right)^{-1}\right)
$$




$$
=\zeta^{n(n-1) / 2} \prod_{i<j} \chi\left(g_{j}, g_{i}\right)^{-1}=(-1)^{n-1} \prod_{i<j} \chi\left(g_{j}, g_{i}\right)^{-1} .
$$

Using Lemma 2.2 we get for all $\sigma \in S_{n}$

$$
\begin{aligned}
\rho(\sigma, & \left.\left(g_{1}, \ldots, g_{n}\right)\right) \prod_{i<j} \chi\left(g_{\sigma(j)}, g_{\sigma(i)}\right)^{-1} \\
& =(-1)^{n-1} \rho\left(\sigma,\left(g_{1}, \ldots, g_{n}\right)\right) \rho\left(\tau,\left(g_{\sigma(1)}, \ldots, g_{\sigma(n)}\right)\right) \\
& =(-1)^{n-1} \rho\left(\sigma \tau,\left(g_{1}, \ldots, g_{n}\right)\right) .
\end{aligned}
$$

Thus we have

$$
\begin{aligned}
S\left(\left[x_{1}, \ldots, x_{n}\right]\right) & =-\overline{\left[x_{1}, \ldots, x_{n}\right]}=-\left[\bar{x}_{1}, \ldots, \bar{x}_{n}\right] \\
& =-\sum_{\sigma \in S_{n}} \rho\left(\sigma,\left(g_{1}, \ldots, g_{n}\right)\right) \bar{x}_{\sigma(1)} \ldots \bar{x}_{\sigma(n)} \\
& =-\sum_{\sigma \in S_{n}} \rho\left(\sigma,\left(g_{1}, \ldots, g_{n}\right)\right)\left(\prod_{i<j} \chi\left(g_{\sigma(j)}, g_{\sigma(i)}\right)^{-1}\right) \bar{x}_{\sigma(n)} \circ \ldots \circ \bar{x}_{\sigma(1)} \\
& =(-1)^{n} \sum_{\sigma \in S_{n}} \rho\left(\sigma \tau,\left(g_{1}, \ldots, g_{n}\right)\right) \bar{x}_{\sigma(n)} \circ \ldots \circ \bar{x}_{\sigma(1)} \\
& =(-1)^{n} \sum_{\sigma \in S_{n}} \rho\left(\sigma,\left(g_{1}, \ldots, g_{n}\right)\right) \bar{x}_{\sigma(1)} \circ \ldots \circ \bar{x}_{\sigma(n)} \\
& =\left[-\bar{x}_{1}, \ldots,-\bar{x}_{n}\right]^{o p}=\left[S\left(x_{1}\right), \ldots, S\left(x_{n}\right)\right]^{o p} .
\end{aligned}
$$

Thus $S$ can be extended to an algebra homomorphism $S: U(P) \longrightarrow U(P)^{o p}$.

We show now that $S$ is the antipode for the bialgebra $U(P)$. Let $x \in P_{h}, x_{i} \in P_{g_{i}}$. We observe first that

$$
S\left(\overline{x x}_{1} \ldots \bar{x}_{n}\right)=S(\bar{x}) \circ S\left(\bar{x}_{1} \ldots \bar{x}_{n}\right)=\chi\left(h, g_{1}+\ldots+g_{n}\right) S\left(\bar{x}_{1} \ldots \bar{x}_{n}\right) S(\bar{x})
$$

since $S$ is a $(G, \chi)$-algebra homomorphism. We now prove $\nabla(1 \otimes S) \Delta(a)=\varepsilon(a)$ for homogeneous terms $a=1, a=\bar{x}$ resp. $a=\bar{x}_{1} \ldots \bar{x}_{n}$ in $U(P)$ by induction.

$$
\begin{gathered}
\nabla(1 \otimes S) \Delta(1)=1 S(1)=1=\varepsilon(1), \\
\nabla(1 \otimes S) \Delta(\bar{x})=\bar{x}+S(\bar{x})=0=\varepsilon(\bar{x}) .
\end{gathered}
$$

Since we can write $\Delta(a)=\sum_{i} a_{i, 1} \otimes a_{i, 2}$ with each of the terms $a_{i, j}$ homogeneous and $\operatorname{deg}\left(a_{i, 1}\right)+\operatorname{deg}\left(a_{i, 2}\right)=\operatorname{deg}(a)(U(P)$ is a $k G$-comodule coalgebra) we get

$$
\begin{aligned}
\nabla(1 \otimes S) \Delta(x a) & =\nabla(1 \otimes S)(x \otimes 1+1 \otimes x)\left(\sum a_{i, 1} \otimes a_{i, 2}\right) \\
& =\nabla(1 \otimes S)\left(\sum x a_{i, 1} \otimes a_{i, 2}+\sum \chi\left(h, \operatorname{deg}\left(a_{i, 1}\right)\right) a_{i, 1} \otimes x a_{i, 2}\right) \\
& =\sum x a_{i, 1} S\left(a_{i, 2}\right)+\sum \chi\left(h, \operatorname{deg}\left(a_{i, 1}\right)\right) a_{i, 1} S\left(x a_{i, 2}\right) \\
& =0+\sum \chi\left(h, \operatorname{deg}\left(a_{i, 1}\right)\right) \chi\left(h, \operatorname{deg}\left(a_{i, 2}\right)\right) a_{i, 1} S\left(a_{i, 2}\right) S(x) \\
& =\chi(h, \operatorname{deg}(a)) \sum a_{i, 1} S\left(a_{i, 2}\right) S(x) \\
& =0=\varepsilon(x a) .
\end{aligned}
$$

Analogously we get $\nabla(S \otimes 1) \Delta=\varepsilon$, so that $S$ is an antipode and $U(P)$ is a $(G, \chi)$-Hopf algebra. 
We come to an interesting consequence for a universal enveloping algebra. There is a new multiplication on $U(P)$ defined by $a \cdot b:=\chi(h, g) \chi(g, h) a b$ for $a, b$ homogeneous with $\operatorname{deg}(a)=h, \operatorname{deg}(b)=g$. This algebra is $U(P)^{o p}$ op which in general will be different from $U(P)$. The map $P \ni x \mapsto \bar{x} \in U(P)^{o p}$ op is a Lie homomorphism (similar to the proof that $S$ was a Lie homomorphism). Thus it induces a homomorphism $U(P) \longrightarrow U(P)^{o p}$ op . Since $U(P)^{o p o p}$ is also generated by $P$ it is easy to see, that it is also a universal enveloping algebra hence $U(P)$ and $U(P)^{o p}$ op are isomorphic under the given homomorphism.

Corollary 4.7. The functor $U:(G, \chi)$-Lie $\longrightarrow(G, \chi)$-Hopf is left adjoint to the functor $P:(G, \chi)$-Hopf $\longrightarrow(G, \chi)$-Lie.

Now we give some examples of braided monoidal categories of $G$-graded modules together with the associated $(G, \chi)$-Lie structures induced by associative algebras. Let $k$ be an algebraically closed field of characteristic zero.

EXAmple 4.8. (1) Let $G=\{0\}$. Then we have $\chi(0,0)=1$ and hence $\chi(0,0) \chi(0,0)$ $=1=(-1)^{2}$ so that $(0,0)$ is a $(-1)$-family. Thus we have a bracket multiplication

$$
[., .]: P_{0} \otimes P_{0} \longrightarrow P_{0} \text {, }
$$

which for associative algebras $A$ is defined by

$$
[x, y]=x y-y x .
$$

This is the example of ordinary Lie algebras. The Lie identities of Definition 4.1 reduce to

$$
\begin{gathered}
{\left[x_{1}, x_{2}\right]=\rho((2,1),(0,0))\left[x_{2}, x_{1}\right]=-\left[x_{2}, x_{1}\right],} \\
\sum_{i=1}^{3} \rho((i \ldots 1),(0,0,0))\left[x_{i},\left[x_{1}, \ldots, \hat{x}_{i}, \ldots, x_{3}\right]\right]= \\
=\left[x_{1},\left[x_{2}, x_{3}\right]\right]+\rho((2,1),(0,0,0))\left[x_{2},\left[x_{1}, x_{3}\right]\right]+\rho((3,2,1),(0,0,0))\left[x_{3},\left[x_{1}, x_{2}\right]\right] \\
=\left[x_{1},\left[x_{2}, x_{3}\right]\right]-\left[x_{2},\left[x_{1}, x_{3}\right]\right]+\left[x_{3},\left[x_{1}, x_{2}\right]\right]=0,
\end{gathered}
$$

and

$$
\left[x,\left[y_{1}, y_{2}\right]\right]=\left[\left[x, y_{1}\right], y_{2}\right]+\chi(0,0)\left[y_{1},\left[x, y_{2}\right]\right]=\left[\left[x, y_{1}\right], y_{2}\right]+\left[y_{1},\left[x, y_{2}\right]\right] \text {. }
$$

(2) Let $G=\mathbf{Z} / 2 \mathbf{Z}=\{0,1\}$ with the bicharacter $\chi(i, j)=(-1)^{i j}$. We have $\chi(0, i)=1$ and hence $\chi(0,1) \chi(1,0)=1=(-1)^{2}$ so the following are $(-1)$-families: $(0,0),(0,1)$, and $(1,0)$ that induce bracket multiplications

$$
\begin{aligned}
& {[., .]: P_{0} \otimes P_{0} \longrightarrow P_{0},} \\
& {[., .]: P_{0} \otimes P_{1} \longrightarrow P_{1},} \\
& {[., .]: P_{1} \otimes P_{0} \longrightarrow P_{1},}
\end{aligned}
$$

which for associative algebras $A$ are defined by

$$
[x, y]=x y-y x \text {. }
$$

Furthermore we have $\chi(1,1)=-1$ and hence $\chi(1,1) \chi(1,1)=1=(-1)^{2}$ so $(1,1)$ is another $(-1)$-family that induces a bracket multiplication

$$
[., .]: P_{1} \otimes P_{1} \longrightarrow P_{0},
$$


with

$$
[x, y]=x y+\rho((2,1),(1,1)) y x=x y+y x .
$$

This is the example of Lie super algebras. The Lie identities of Definition 4.1 reduce to

$$
\begin{gathered}
{\left[x_{1}, x_{2}\right]=\rho\left((2,1),\left(\operatorname{deg}\left(x_{1}\right), \operatorname{deg}\left(x_{2}\right)\right)\right)\left[x_{2}, x_{1}\right]=-(-1)^{\operatorname{deg}\left(x_{1}\right) \operatorname{deg}\left(x_{2}\right)}\left[x_{2}, x_{1}\right]} \\
{\left[x_{1},\left[x_{2}, x_{3}\right]\right]-(-1)^{\operatorname{deg}\left(x_{1}\right) \operatorname{deg}\left(x_{2}\right)}\left[x_{2},\left[x_{1}, x_{3}\right]\right]} \\
+(-1)^{\left(\operatorname{deg}\left(x_{1}\right)+\operatorname{deg}\left(x_{2}\right)\right) \operatorname{deg}\left(x_{3}\right)}\left[x_{3},\left[x_{1}, x_{2}\right]\right]=0 \\
{\left[x,\left[y_{1}, y_{2}\right]\right]=\left[\left[x, y_{1}\right], y_{2}\right]+(-1)^{\operatorname{deg}(x) \operatorname{deg}\left(y_{1}\right)}\left[y_{1},\left[x, y_{2}\right]\right]}
\end{gathered}
$$

(3) Let $G$ be an arbitrary abelian group with a bicharacter $\chi$ such that $\chi\left(g_{1}, g_{2}\right)=$ $\chi\left(g_{2}, g_{1}\right)^{-1}$ for all $g_{1}, g_{2} \in G$. Then we have $\chi\left(g_{1}, g_{2}\right) \chi\left(g_{2}, g_{1}\right)=1=(-1)^{2}$. This defines $(-1)$-families $\left(g_{1}, g_{2}\right)$ together with bracket operations

$$
\begin{gathered}
{[., .]: P_{g_{1}} \otimes P_{g_{2}} \longrightarrow P_{g_{1}+g_{2}},} \\
{[x, y]=x y+\rho\left((2,1),\left(g_{1}, g_{2}\right)\right) y x=x y-\chi\left(g_{1}, g_{2}\right) y x .}
\end{gathered}
$$

This is the example of Lie color algebras for an abelian group. The Lie identities of Definition 4.1 reduce to

$$
\begin{gathered}
{\left[x_{1}, x_{2}\right]=\rho\left((2,1),\left(\operatorname{deg}\left(x_{1}\right), \operatorname{deg}\left(x_{2}\right)\right)\right)\left[x_{2}, x_{1}\right]=-\chi\left(\operatorname{deg}\left(x_{1}\right), \operatorname{deg}\left(x_{2}\right)\right)\left[x_{2}, x_{1}\right],} \\
{\left[x_{1},\left[x_{2}, x_{3}\right]\right]-\chi\left(\operatorname{deg}\left(x_{1}\right), \operatorname{deg}\left(x_{2}\right)\right)\left[x_{2},\left[x_{1}, x_{3}\right]\right]} \\
+\chi\left(\operatorname{deg}\left(x_{1}\right)+\operatorname{deg}\left(x_{2}\right), \operatorname{deg}\left(x_{3}\right)\right)\left[x_{3},\left[x_{1}, x_{2}\right]\right]=0, \\
{\left[x,\left[y_{1}, y_{2}\right]\right]=\left[\left[x, y_{1}\right], y_{2}\right]+\chi\left(\operatorname{deg}(x), \operatorname{deg}\left(y_{1}\right)\right)\left[y_{1},\left[x, y_{2}\right]\right] .}
\end{gathered}
$$

(4) Let $G=\mathbf{Z} / 3 \mathbf{Z}=\{0,1,2\}$ with the bicharacter $\chi(i, j)=\zeta^{i j}$ where $\zeta$ is a primitive 3 rd root of unity. Then $\chi(0, i) \chi(i, 0)=1=(-1)^{2}$ so that we get $(-1)$-families $(0, i)$ with

$$
\begin{gathered}
{[., .]: P_{0} \otimes P_{i} \longrightarrow P_{i},} \\
{[., .]: P_{i} \otimes P_{0} \longrightarrow P_{i},} \\
{[x, y]=x y-y x .}
\end{gathered}
$$

Furthermore we have $\chi(i, j) \chi(j, i)=\left(\zeta^{i j}\right)^{2}$ with a primitive 3 rd root of unity $\zeta^{i j}$ for all $i, j \neq 0$. This gives $\zeta$-families $(1,1,1)$ and $(2,2,2)$ and no $\zeta^{2}$-family. The associated Lie structure is

$$
[\ldots]: P_{i} \otimes P_{i} \otimes P_{i} \longrightarrow P_{0}
$$

with

$$
\left[x_{1}, x_{2}, x_{3}\right]=\sum_{\sigma \in S_{3}} x_{\sigma(1)} x_{\sigma(2)} x_{\sigma(3)}
$$

since $\rho(\sigma,(i, i, i))=\prod\left(\zeta^{-1} \chi(i, i)\right)=1$ for all $\sigma \in S_{3}$. The Lie identities of Definition 4.1 for these ternary brackets reduce to

$$
\begin{gathered}
{\left[x_{1}, x_{2}, x_{3}\right]=\left[x_{\sigma(1)}, x_{\sigma(2)}, x_{\sigma(3)}\right]} \\
{\left[x_{1},\left[x_{2}, x_{3}, x_{4}\right]\right]+\left[x_{2},\left[x_{1}, x_{3}, x_{4}\right]\right]+\left[x_{3},\left[x_{1}, x_{2}, x_{4}\right]\right]+\left[x_{4},\left[x_{1}, x_{2}, x_{3}\right]\right]=0,}
\end{gathered}
$$


for $x_{1}, x_{2}, x_{3}, x_{4} \in P_{i}, i=1$ or $i=2$. Here we also find a first example where the two Jacobi identities mean different things. The second Jacobi identity for $x \in P_{0}$ and $y_{1}, y_{2}, y_{3} \in P_{i}, i=1$ or $i=2$ reduces to

$$
\left[x,\left[y_{1}, y_{2}, y_{3}\right]\right]=\left[\left[x, y_{1}\right], y_{2}, y_{3}\right]+\left[y_{1},\left[x, y_{2}\right], y_{3}\right]+\left[y_{1}, y_{2},\left[x, y_{3}\right]\right]
$$

Furthermore there are $(-\zeta)$-families $(1,1,1,1,1,1)$ and $(2,2,2,2,2,2)$ for the primitive 6 -th root of unity $-\zeta$ which give maps $\otimes{ }^{6} P_{1} \rightarrow P_{0}$ and $\otimes{ }^{6} P_{2} \rightarrow P_{0}$. The associated Lie multiplication of an associative algebra is

$$
\left[x_{1}, \ldots, x_{6}\right]=\sum_{\sigma \in S_{6}} \operatorname{sgn}(\sigma) x_{\sigma(1)} \ldots x_{\sigma(6)}
$$

since $\rho(\sigma,(i, i, i, i, i, i))=\prod\left(-\zeta^{-1} \chi(i, i)\right)=\operatorname{sgn}(\sigma)$ for all $\sigma \in S_{6}$.

(5) Let $G=C_{n} \times \ldots \times C_{n}$ (r-times) with generators $t_{1}, \ldots, t_{r}$. Since later we will take the biproduct of a $(G, \chi)$-Hopf algebra with $k G$, we will write $G$ multiplicatively in this example. Let $\chi$ be given by $\chi\left(t_{i}, t_{j}\right)=\zeta$, a primitive $n$-th root of unity. Then $\left(t_{1}, \ldots, t_{1}\right)$ is a $\zeta$-family. $\mathrm{A}(G, \chi)$-Lie algebra will have a bracket operation

$$
[., .]: \otimes^{n} P_{t_{1}} \longrightarrow P_{0}
$$

with

$$
\left[x_{1}, \ldots, x_{n}\right]=\sum_{\sigma \in S_{n}} x_{\sigma(1)} \ldots x_{\sigma(n)} .
$$

(6) The last example of this kind comes with $G=\mathbf{Z} / 3 \mathbf{Z} \times \mathbf{Z} / 3 \mathbf{Z}$ and generators $g_{1}, g_{2}$ each of order 3. Define $\chi$ by $\chi\left(g_{1}, g_{1}\right):=\zeta$, a primitive 3rd root of unity, $\chi\left(g_{1}, g_{2}\right):=$ $\zeta^{2}, \chi\left(g_{2}, g_{1}\right)=1$, and $\chi\left(g_{2}, g_{2}\right)=\zeta$. Then there are several $\zeta$-families, among others $\left(g_{1}, g_{1}, g_{2}\right)$ and $\left(g_{1}, g_{2}, g_{2}\right)$. They define brackets

$$
\begin{aligned}
& {[\ldots]: P_{g_{1}} \otimes P_{g_{1}} \otimes P_{g_{2}} \longrightarrow P_{2 g_{1}+g_{2}},} \\
& {[\ldots]: P_{g_{1}} \otimes P_{g_{2}} \otimes P_{g_{2}} \longrightarrow P_{g_{1}+2 g_{2}},}
\end{aligned}
$$

with

$$
\begin{aligned}
{\left[x_{1}, x_{2}, x_{3}\right] } & =\sum_{\sigma \in S_{3}} \rho\left(\sigma,\left(g_{1}, g_{1}, g_{2}\right)\right) x_{\sigma(1)} x_{\sigma(2)} x_{\sigma(3)} \\
& =x_{1} x_{2} x_{3}+x_{2} x_{1} x_{3}+\zeta x_{1} x_{3} x_{2}+\zeta x_{2} x_{3} x_{1}+\zeta^{2} x_{3} x_{1} x_{2}+\zeta^{2} x_{3} x_{2} x_{1}
\end{aligned}
$$

resp.

$$
\begin{aligned}
{\left[x_{1}, x_{2}, x_{3}\right] } & =\sum_{\sigma \in S_{3}} \rho\left(\sigma,\left(g_{1}, g_{1}, g_{2}\right)\right) x_{\sigma(1)} x_{\sigma(2)} x_{\sigma(3)} \\
& =x_{1} x_{2} x_{3}+x_{1} x_{3} x_{2}+\zeta x_{2} x_{1} x_{3}+\zeta x_{3} x_{1} x_{2}+\zeta^{2} x_{2} x_{3} x_{1}+\zeta^{2} x_{3} x_{2} x_{1}
\end{aligned}
$$

We close with some examples of $(G, \chi)$-Lie algebras and of $(G, \chi)$-Hopf algebras generated by them. We also examine some of the biproduct Hopf algebras one always obtains from $(G, \chi)$-Hopf algebras. If $H$ is a $(G, \chi)$-Hopf algebra then $H \otimes k G$ is a Hopf algebra by

$$
\begin{gathered}
(x \otimes g)(y \otimes h)=\chi(g, \operatorname{deg}(y)) x y \otimes g h, \\
\Delta(1 \otimes g)=(1 \otimes g) \otimes(1 \otimes g),
\end{gathered}
$$


and

$$
\Delta(x \otimes 1)=\sum_{a+b=c}\left(y_{a, i} \otimes b\right) \otimes\left(z_{b, i} \otimes 1\right),
$$

where $\Delta(x)=\sum_{a+b=c} \sum_{i} y_{a, i} \otimes z_{b, i}$ with $\operatorname{deg}(x)=c, \operatorname{deg}\left(y_{a, i}\right)=a$, and $\operatorname{deg}\left(z_{b, i}\right)=b$ (see $[\mathrm{FM}]$ Corollary 3.5).

Most of the $(G, \chi)$-Hopf algebras are infinite-dimensional. In fact, the only finitedimensional Hopf algebras generated by their primitives, we know, are given in the following example under (1) and (2).

ExAmple 4.9. (1) $P=P_{1}=k x$ with $[x, x]=0$ defines a "commutative" $\left(C_{2}, \chi\right)$ Lie algebra with $\chi$ as in Example 4.8. (2). It generates the $\left(C_{2}, \chi\right)$-Hopf algebra $H=$ $k[x] /\left(x^{2}\right)$, the universal enveloping algebra of $P$. The biproduct $H \star k C_{2}$ is the well known smallest noncommutative noncocommutative Hopf algebra ([FM] Example 3.9).

(2) Let $(G, \chi)$ be as in Example $4.8(5) . P=P_{t_{1}}=k x$ with $[x, \ldots, x]=0$ defines a "commutative" $(G, \chi)$-Lie algebra with $\chi\left(t_{1}, t_{1}\right)=\zeta$, a primitive $n$-th root of unity. It generates the $(G, \chi)$-Hopf algebra $H=k[x] /\left(x^{n}\right)$, given in the introduction (for $r=1$ ). The biproduct $U(P) \star k G$ is the free algebra generated by the elements $x, t_{1}, \ldots, t_{r}$ subject to the following relations $t_{i}^{n}=1, t_{i} t_{j}=t_{j} t_{i}$, and $x^{n}=0$. There is one more set of relations that is obtained from the multiplicative rule for the biproduct $\left(1 \otimes t_{i}\right)(x \otimes 1)=\chi\left(t_{i}, t_{1}\right) x \otimes t_{i}$ or - identifying $x \otimes 1$ with $x$ and $1 \otimes t_{i}$ with $t_{i}$ - the relations $t_{i} x=\zeta x t_{i}$. Hence the biproducts form the family of noncommutative noncocommutative Hopf algebras given in $[\mathrm{T}]$.

(3) $P=\bigoplus_{i=0}^{2} P_{i}, P_{0}=k z, P_{1}=k x \oplus k y, P_{2}=0$ with $\left([]:, \otimes{ }^{6} P_{1} \rightarrow 1\right)=0$ and $[x, x, x]=[y, y, y]=[x, y, y]=0,[x, x, y]=z$, and $[z, x]=[z, y]=0$ define a $\left(C_{3}, \chi\right)$-Lie algebra with $\chi(\overline{1}, \overline{1})=\zeta$, a primitive 3 rd root of unity. It generates the $\left(C_{3}, \chi\right)$-Hopf algebra $H=k\langle x, y\rangle /\left(x^{3}, y^{3}, x y^{2}+y x y+y^{2} x\right)$ (we have $\left.z=2\left(x^{2} y+x y x+y x^{2}\right)\right)$, the universal enveloping algebra of $P$. The biproduct $U(P) \star k C_{3}$ has generators $x, y, t$ with the relations $x^{3}=y^{3}=0, t^{3}=1, x y^{2}+y x y+y^{2} x=0, x t=\zeta t x$, and $y t=\zeta t y$.

(4) Let $G=C_{3}$, and $\chi$ be as before. Then $P=\bigoplus_{i=0}^{2} P_{i}, P_{0}=k y, P_{1}=k x, P_{2}=0$ with $[x, x, x, x, x, x]=0,[x, x, x]=y$, and $[y, x]=0$ defines a $\left(C_{3}, \chi\right)$-Lie algebra. It generates the $\left(C_{3}, \chi\right)$-Hopf algebra $H=k[x, y] /\left(y-6 x^{3}\right)=k[x]$, the universal enveloping algebra of $P$.

(5)For $G, \chi$ as before let $P_{0}=k y, P_{1}=k x$, and $P_{2}=0$ with $[x, x, x, x, x, x]=0$, $[x, x, x]=0$ and $[y, x]=x$. Then $P$ is a $\left(C_{3}, \chi\right)$-Lie algebra. It generates the $\left(C_{3}, \chi\right)$-Hopf algebra $H=k\langle x, y\rangle /\left(x^{3}, x-y x+x y\right)$, the universal enveloping algebra of $P$.

\section{References}

[A] George E. Andrews, The Theory of Partitions, Encyclopedia of Mathematics and its Applications. Vol. 2, Addison-Wesley, 1976.

[FM] Davida Fischman and Susan Montgomery, A Schur Double Centralizer Theorem for Cotriangular Hopf Algebras and Generalized Lie Algebras, J. Algebra 168 (1994), 594-614. 
[M94a] Shahn Majid, Crossed Products by Braided Groups and Bosonization, J. Algebra 163 (1994), 165-190.

[M94b] Shahn Majid, Algebra and Hopf Algebras in Braided Categories, in: Advances in Hopf Algebras. LN pure and applied mathematics 158 (1994) 55-105.

[R] David Radford, The Structure of Hopf Algebras with a Projection, J. Algebra 92 (1985), 322-347.

[T] Earl J. Taft, The Order of the Antipode of Finite-dimensional Hopf algebras. Proc. Nat. Acad. Sci. USA 68 (1971), 2631-2633. 